\title{
Research Paper \\ The Effects of Eight Weeks Selected Combined Exercises on Humoral Immune and Hema- tological Index in Inactive Older Men
}

\author{
Ehsan Mir ${ }^{1}, *$ Seyyed Reza Attarzadeh Hosseini ${ }^{1}$, Mojtaba Mir Sayeedi ${ }^{2}$, Keyvan Hejazi ${ }^{1}$
}

Citation: Mir E, Attarzadeh Hosseini SR, Mir Sayeedi M, Hejazi K. [The effects of eight weeks selected combined exercises on humoral immune and hematological index in inactive older men (Persian)]. Iranian Journal of Ageing. 2016; 11(4):20-29. http://dx.doi.org/10.21859/sija-110120

http://dx.doi.org/10.21859/sija-110120

Received: 16 Dec. 2015 Accepted: 18 Jan. 2016

Key words: Immunoglobulin, Training, Elderly

\begin{abstract}
Objectives Old age is associated with irregularities in many aspects of body immune system function. As sports activities could affect the immune system and old age is associated with progressive immune failure, the study of the effects of exercise on the immune system function in old age becomes important. Therefore, this study aimed to investigate the effects of selected combined exercises (aerobic and resistance training) on the serum level of cortisol and immunoglobulins in inactive elderly men.

Methods \& Materials In this quasi-experimental study, 24 subjects were selected by convenience sampling method. Their age and body mass index ranged $60-70$ years and $22-25 \mathrm{~kg} / \mathrm{m} 2$, respectively. Then, they were randomly assigned into 2 groups (experimental [n=12] and control [n=12]). The experimental group started the combined training exercise, and the control group continued their inactive usual routines. The combined training exercise (aerobic-resistance) included running on a treadmill for 20 minutes per session, 3 sessions per week, for 8 weeks, with an intensity of $60 \%$ to $70 \%$ HRR. Furthermore, the resistance training comprised 10 circling stationary movements of leg flexion, leg extension, leg press, scott, underarm stretch, chest press, iron cross with dumbbells, biceps flexion, triceps extension, and rowing motion with rope. This training included an intensity of $60 \%$ to $70 \%$ of one maximum repetition with extra load and 10 repetitions in 2 successive times with 30 seconds rest between each repetition and 2 minutes' rest between each movement. In this study, the blood samples were taken 24 hours before the exercise and 24 hours after the last session of the exercise. We used nephelometer instrument and binding kit site to measure the IgG, IgM, and IgA levels. The cortisol level was measured by Gamacounter and RIA kit by the immunoradiometric method. To evaluate the consumed oxygen, we used the protocol on treadmill instrument. In order to compare intra- and inter-group mean variables, we used analyses of variance for repeated measures. The significant level was set at $P<0.05$.

Results The results indicated that the combined exercises reduced IgG level $(12.75 \%)$ in the elderly people $(P=0.04)$. Although the level of serum IgM was also reduced $(15.93 \%)$, this decrease was insignificant $(P=0.31)$. Furthermore, no significant change was seen in the IgA level $(P=0.90)$. The cortisol level was significantly increased $(8.33 \%)$ in the experimental group $(P=0.001)$. Based on the results, the mean intergroup changes in the levels of IgA, IgA, and IgM were not significant $(P>0.05)$. However, a significant difference was seen between 2 groups with regard to serum cortisol level $(P<0.05)$.

Conclusion Doing physical activities is one of the key factors in changing the function of the immune system. This issue is associated with the intensity, duration, kind of exercise, body readiness, nutrition, mental status, and hormonal factors. Based on the results, long and intensive exercises with an increase in the catabolic process may weaken the immune system of the older people.
\end{abstract}




\title{
تأثير هشت هفته تمرينات تركيبى منتخب بر سطوح ايمنى هومورال و شاخصهاى هماتولوزيك مردان سالمند غيرفعال
}

\author{
احسان مير'، "سيدرضا عطارزادهسينى'، مجتبى ميرسعيدى'، كيوان حجازى'

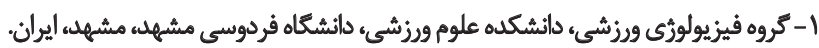

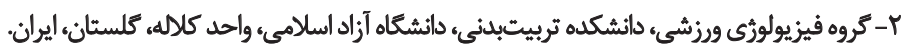

\begin{abstract}
حكبد

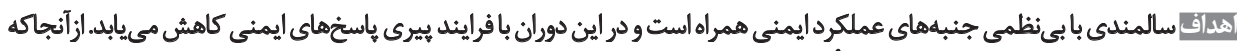

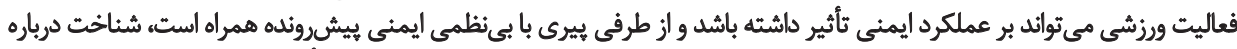

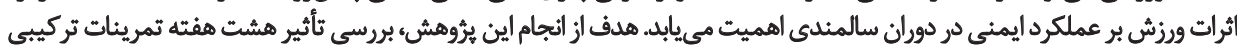

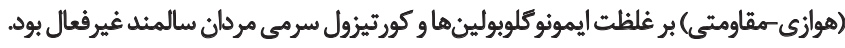

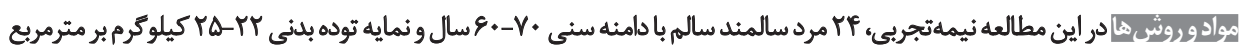

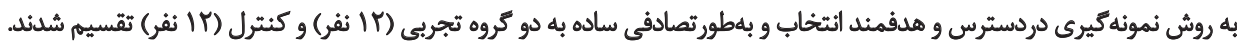

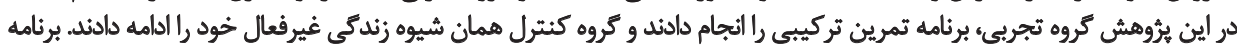

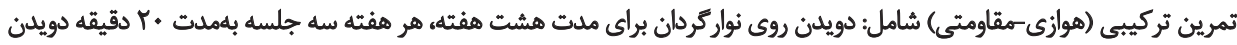

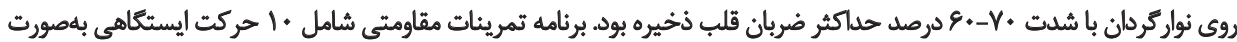

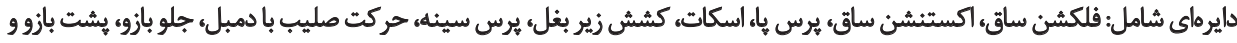

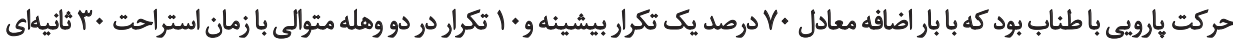

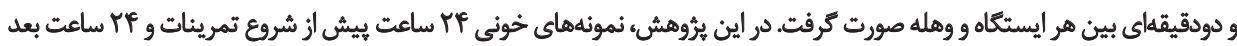

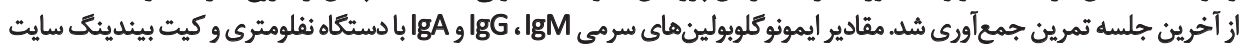

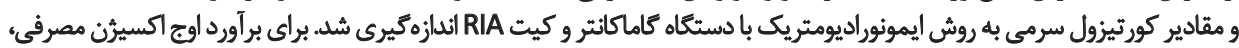

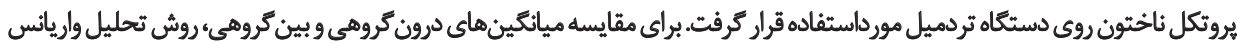

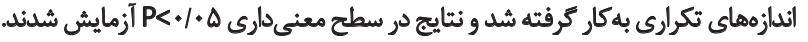

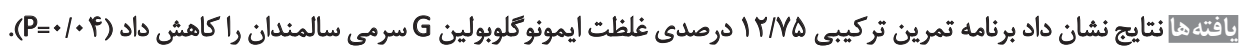

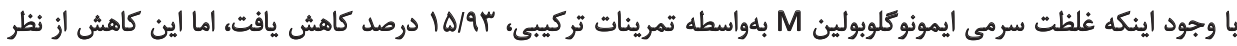

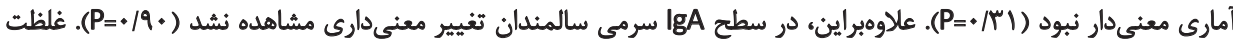

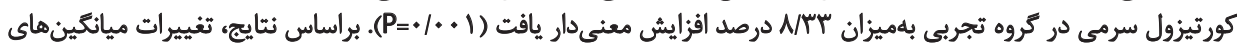

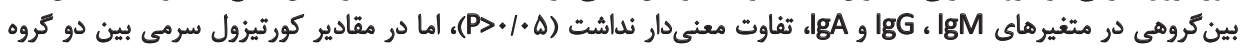

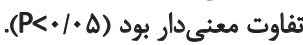

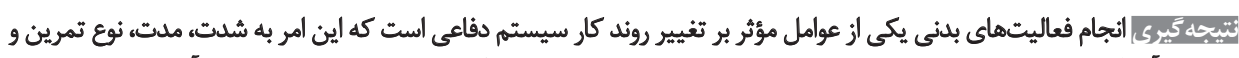

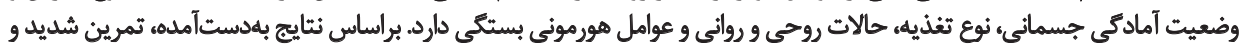

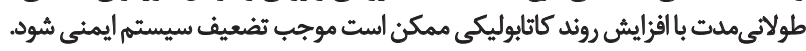

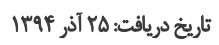

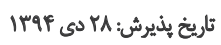

$$
\text { كليدوأوهاها: }
$$

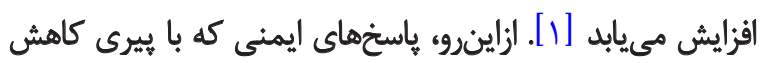

مقلمه

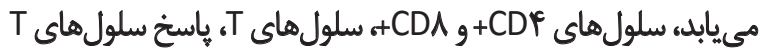

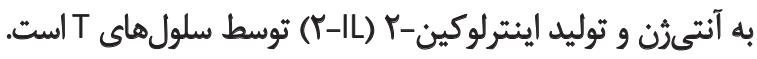

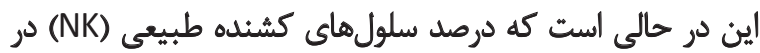

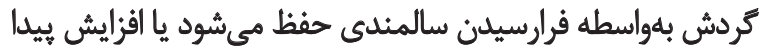

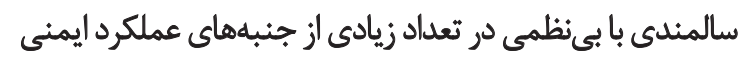

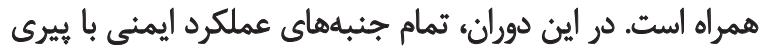

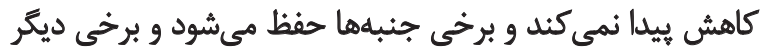

-

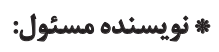

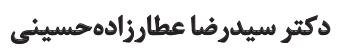

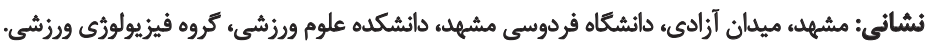

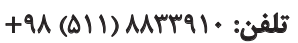

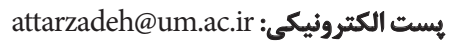




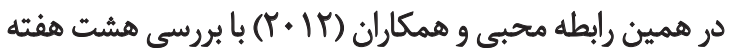

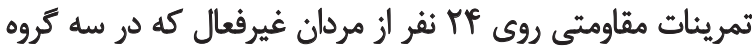

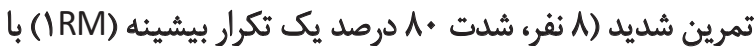

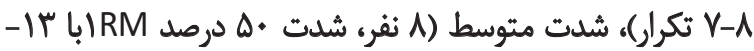

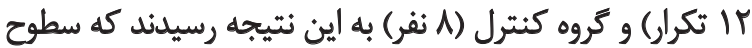

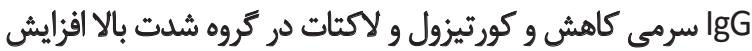

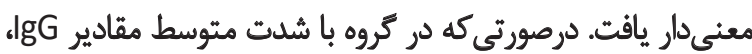
لكوسيتها و كورتيزول كاهش معنى دارى يافت [1 1 ].

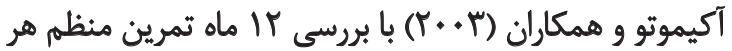

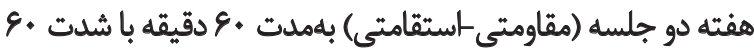

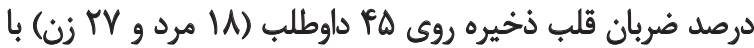

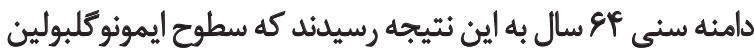

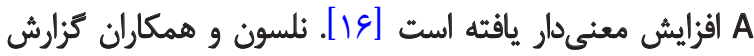

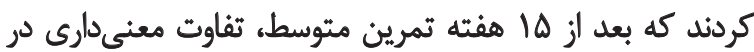

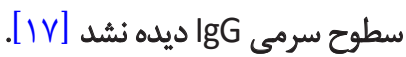

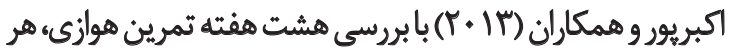

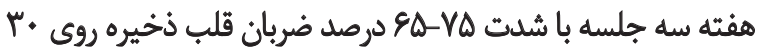

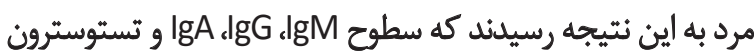

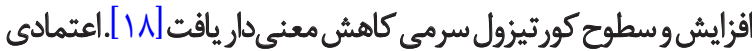

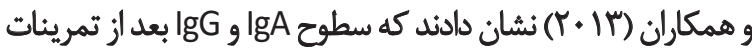

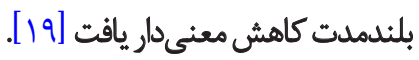

فعاليت ورزشى در دوره سالمندى براى ييشَّيرى از بيمارىهاى

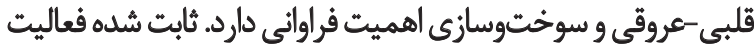

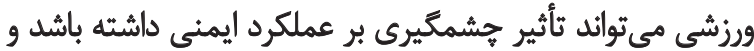

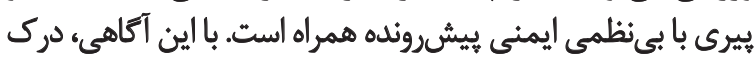

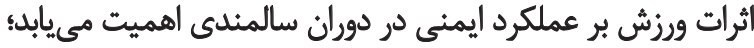

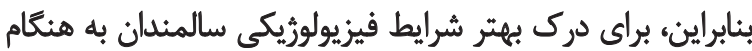

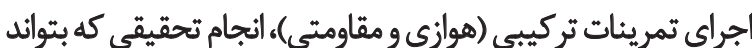

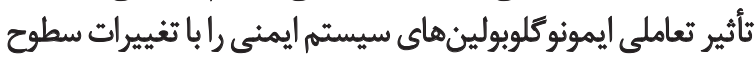

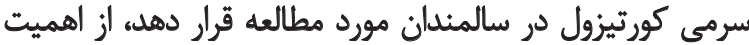

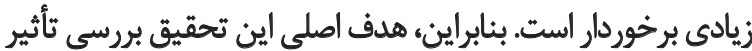

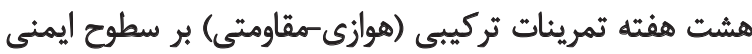

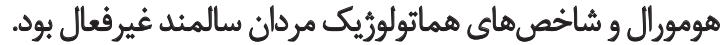

$$
\text { روش مطالعه }
$$

اين تحقيق از نوع نيمهتجبيى است كه دو كروه تجربى با طرح

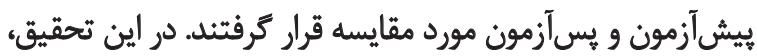

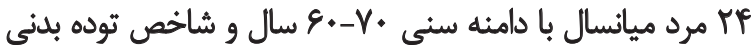

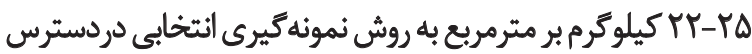

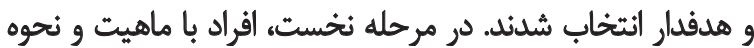

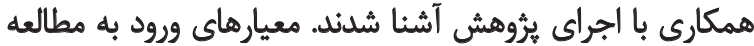

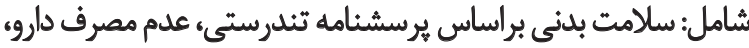

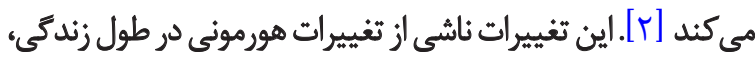
افزايش توليد راديكالهاي آزاد و تجمع رويارويى با با أنتىثنهاست. بإئظمى مرتبط با سن در برخى از جنبههاي عملكرد ايمنى

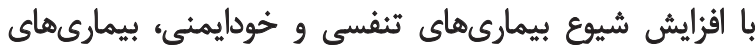

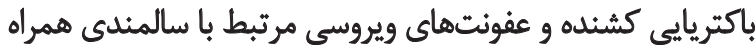

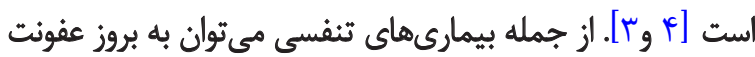

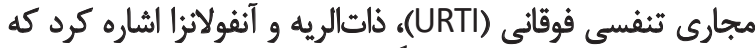

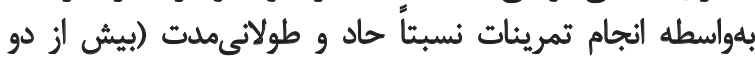

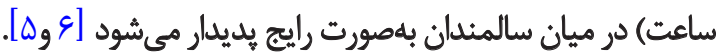
افزايش خطر عفونت ويروسى يا باكتريايى به كاهش يا تغيير

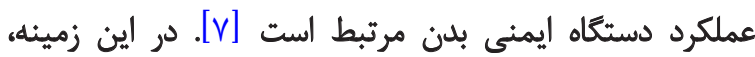

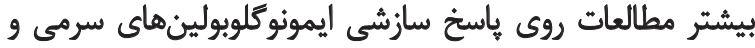

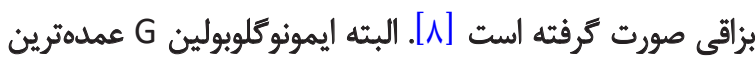

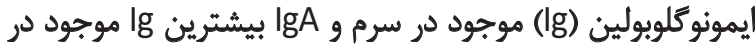

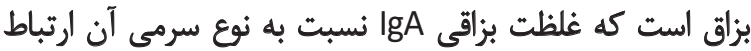

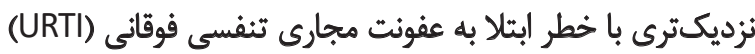

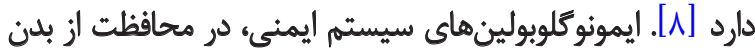

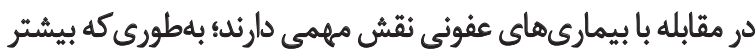

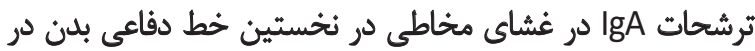

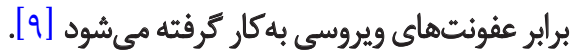
نتيجه بسيارى از ئروهشها نشان مىدهد كه به سبب تغيير در

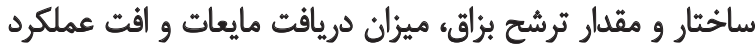

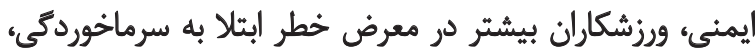

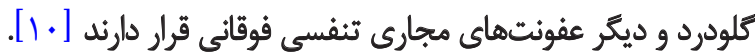

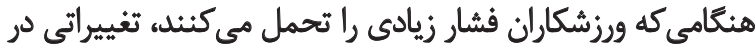

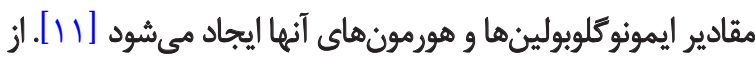

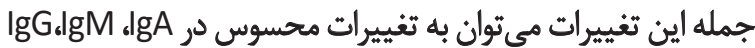

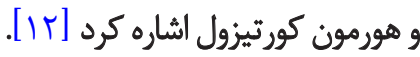

در اين راستا، افزايش غلظت هورمونهاى آدرنالين، كورتيزول،

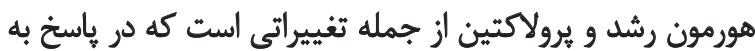

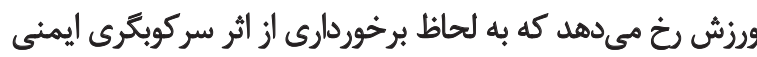

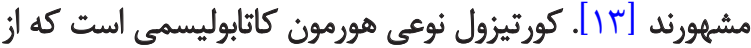

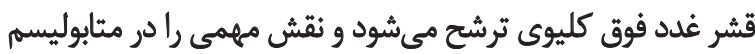

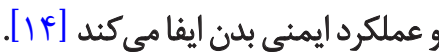

افزايش سطح هورمونهاى استرس از جمله كورتيزول در خون،

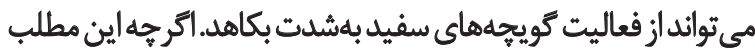

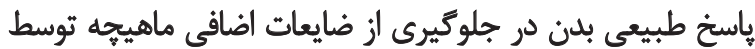

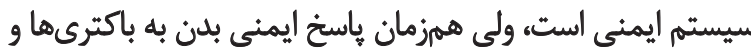

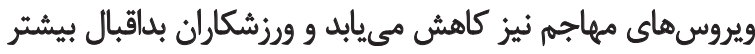

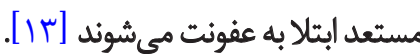


درصد ضربان قلب ذخيره بلوسيله ضربانسنج يولار (ساخت كشور

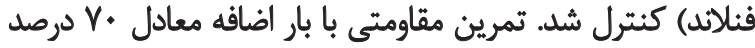

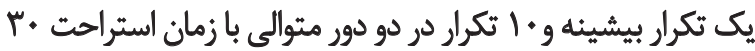

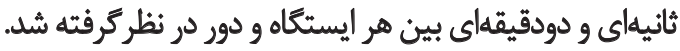
تمرينات مقاومتى شامل · 1 حركت ايستّاهى باصورت دايرهاي

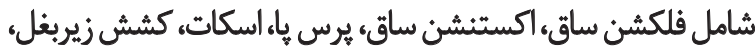

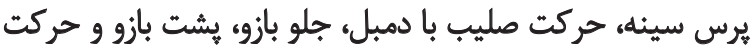

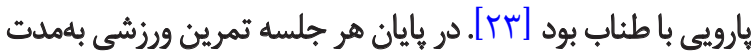

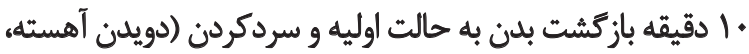

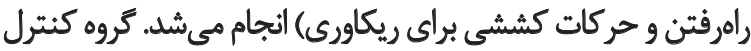

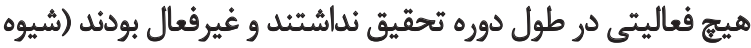

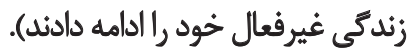

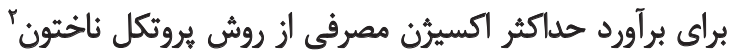

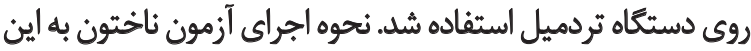

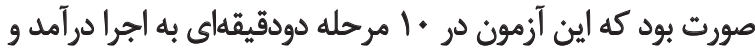

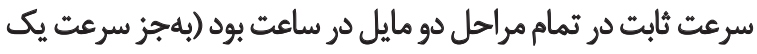

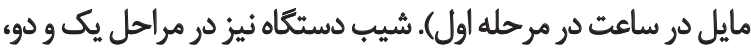

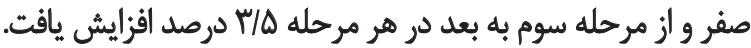
حداكثر اكسيرن مصرفي در يروتكل ناختون با با ستفاده از معادله

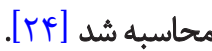

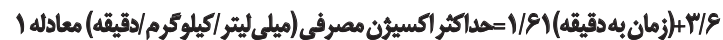

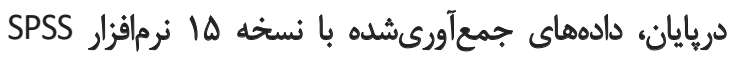

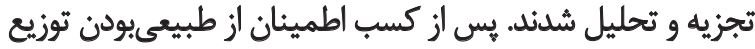

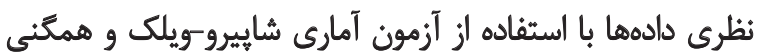

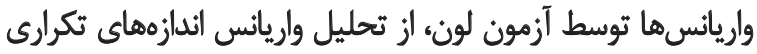

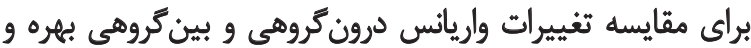
سطح معنى دارى كمتر از هـ ٪+ در نظر كرفته شد.

ياثتهن

مشخصات آزمودنىهاى كروه تجربى و كنترل در جدول شماره

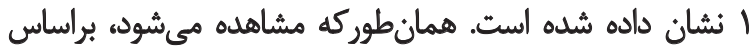

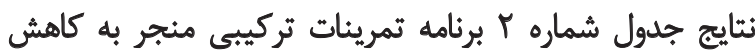

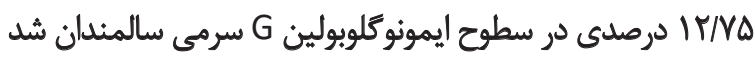

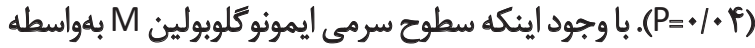

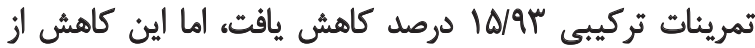

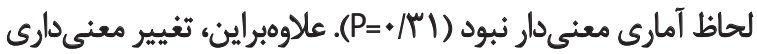

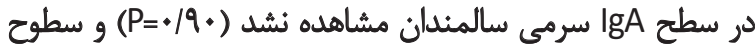

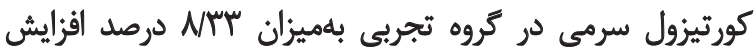

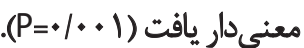
لازم بدذكر است كه تغييرات ميانكينهاى درون كروهى متغيرهاى
عدماستعمال دخانيات و شركتنكردن در هيج برنامه تمرينى حداقل

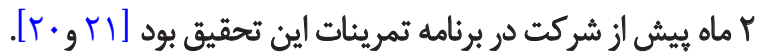
براساس يرسشنامه اطلاعات فردى، سوابق يزٔشكى، معاينه و نظر

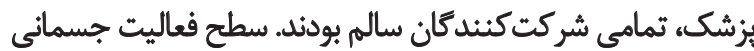

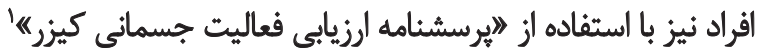

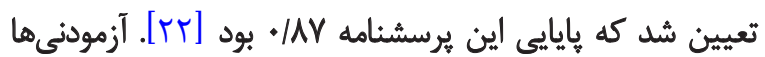

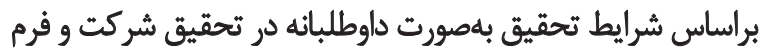

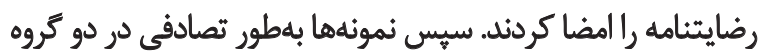

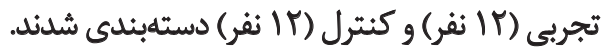

براى ارزيابى ثركيبات بلن بهثرتيب طول قد آزمودنىها با قدسنج

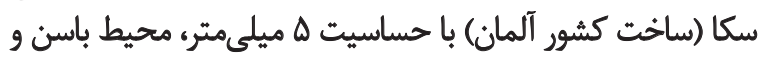

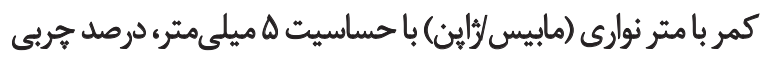

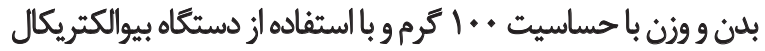

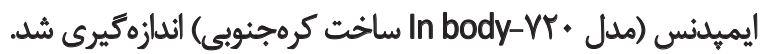

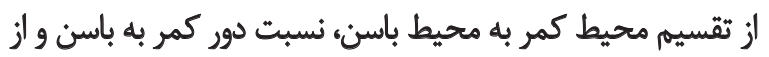

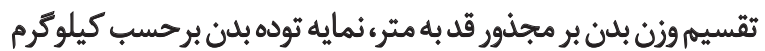
بر مترمربع بهدست آمد.

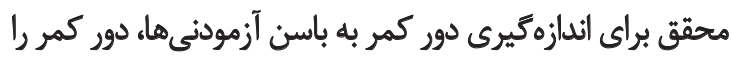

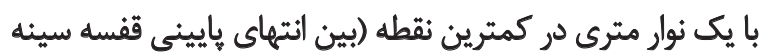

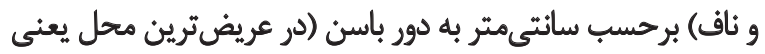

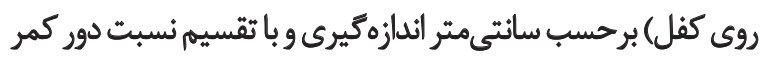

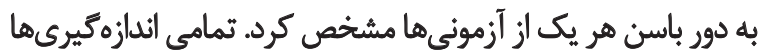

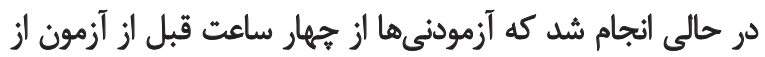

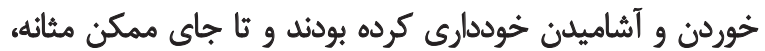

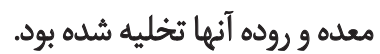

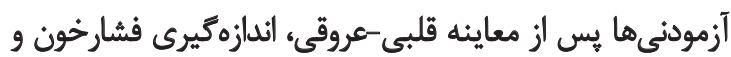

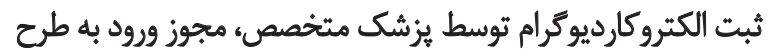

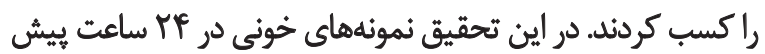

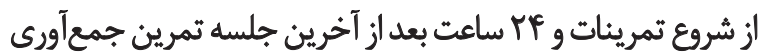

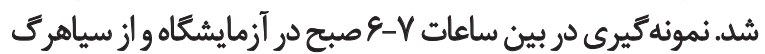

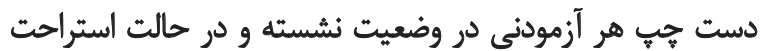

$$
\text { صورت كرفت. }
$$

براى اندازمكيرى خودكار مقادير ايمونوكلوبولينهاى سرمى إنى

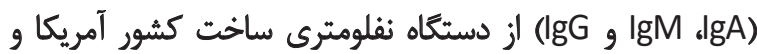

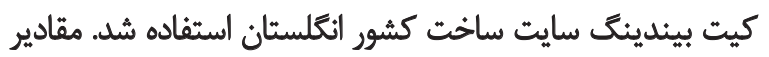

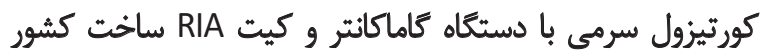

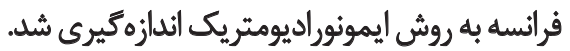

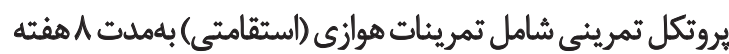

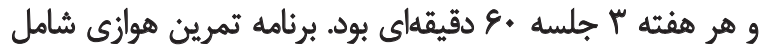

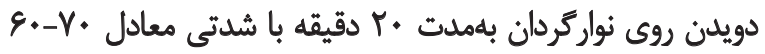

1. Kaiser physical activity survey 


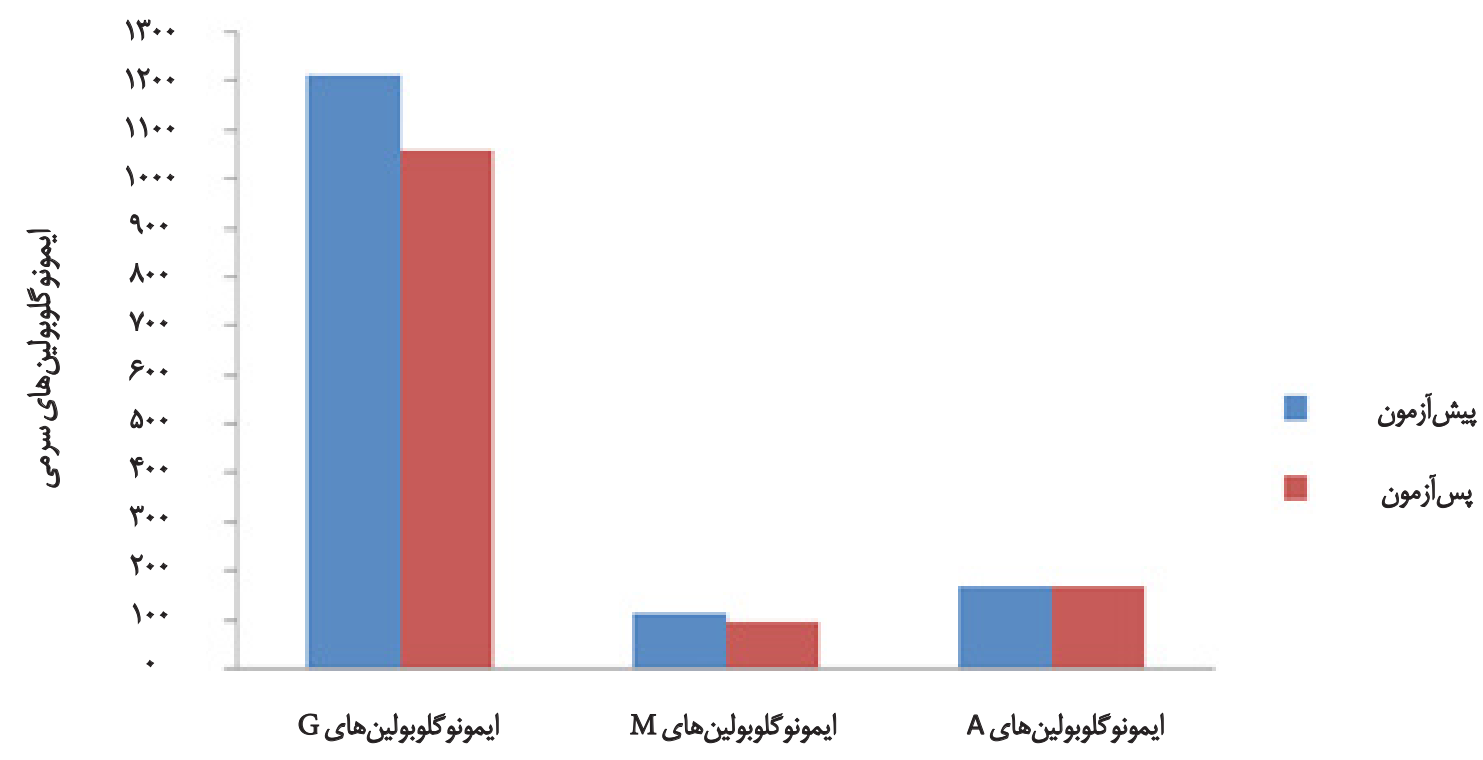

جدول ا. ويرّكى هاى آزمودنىهاى شركت كنئده در مطالعه.

\begin{tabular}{|c|c|c|c|c|}
\hline \multicolumn{4}{|c|}{ (انحراف معياريريائكين) } & \multirow{2}{*}{ كروهها } \\
\hline نمايه توده بدن (كيلوكرم) & (كيلو وزتم) & (متر) & سن & \\
\hline$M e / \Delta \pm Y / F$ & $V^{w} / \mathrm{r} \pm V / \bar{C}$ & $\mid M T \pm \cdot M I$ & $g \in / Y \pm T / K$ & تجنئي \\
\hline$r \times / r \pm ו / /$ & $r / r u \pm r / r$ & WrTI./Me & $R T / Y \pm T / I$ & كنترل \\
\hline
\end{tabular}

次

كه هشت هفته تمرين تركيبى در مردان سالمند، منجر به كاهش

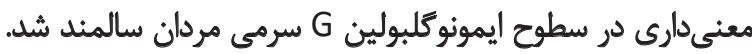

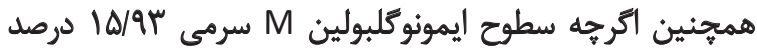

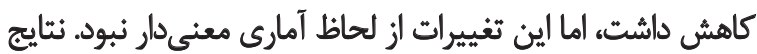

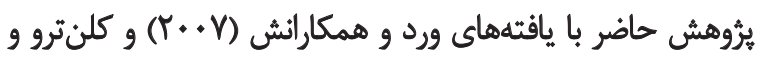

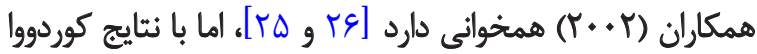

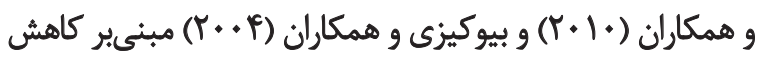

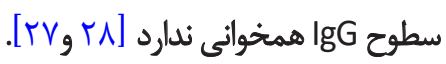

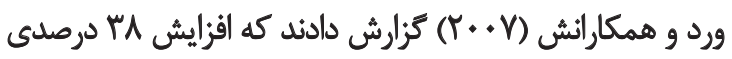

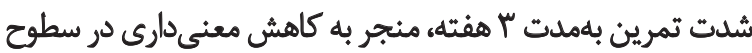

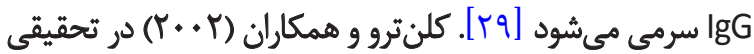
نشان دادند كه سطوح IgG سرمى بهواسطه انجام تمرين شديد

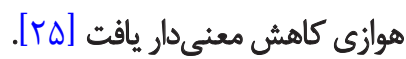
كوردووا و همكاران (•l(T) افزايش معنى دارى را در سطوح

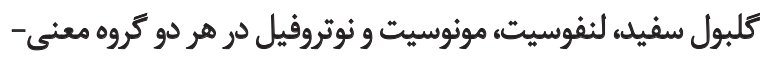

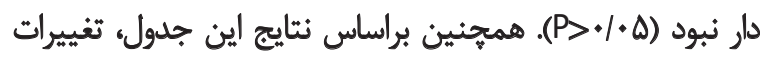

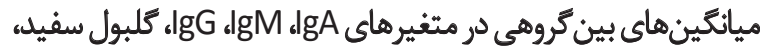

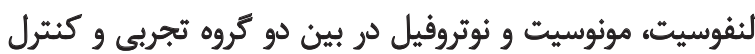

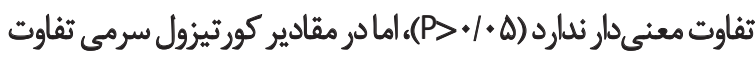

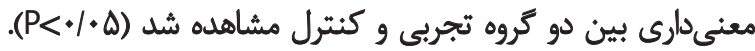

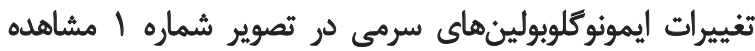

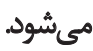
بـ هدف از مطالعه حاضر، بررسى ثأثير هشت هفته تمرينات

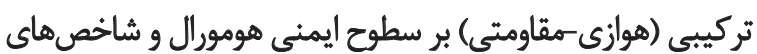

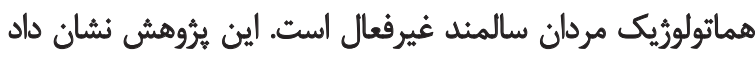


جدول r. مثايسه تغييرات واريانس درون كروهى و بين كروهى در سطوح سرمى IgG IgM ،IgA، كورتيزول و شاخصهاى هماتولوزيكى مردان ميانسال غيرفعال.

\begin{tabular}{|c|c|c|c|c|c|}
\hline \multicolumn{2}{|c|}{ تغييرات } & \multirow{2}{*}{\multicolumn{2}{|c|}{ 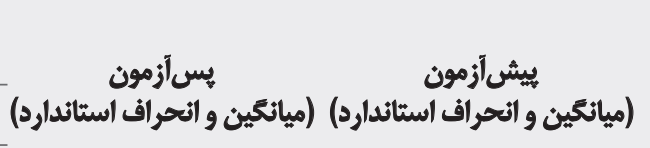 }} & \multirow{3}{*}{$\log _{0}$} & \multirow{3}{*}{ متغيرها } \\
\hline بين كروها & دوونكووه & & & & \\
\hline $\mathrm{P}$-value & $\mathrm{P}$-value & & & & \\
\hline \multirow{2}{*}{.$/ N T$} & $.1 . e^{t}$ & 1. $8 . / N \Delta \pm 1 Y T / T q$ & IYIه/AY"IIQVV. & تجربى & \multirow{2}{*}{ ايمونوكلوبولين G (ميلى كرم اليث) } \\
\hline & $\cdot / A Y$ & $|r \cdot V / 9| \pm M Y N+1$ & Irqq/eg士reg/ro & كثترل & \\
\hline \multirow{2}{*}{. } & $\cdot \mid m$ & $Q 9 / 9 . \pm \Delta F / M T$ & $\|f / q\| \pm r \cdot \mid q r$ & تجريى & \multirow{2}{*}{ 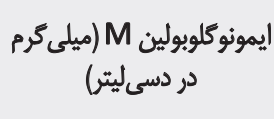 } \\
\hline & $\cdot / M F$ & $1+q / r \mid \varnothing \pm \pi r / \Delta V$ & $q V / 8+ \pm \Delta \& / \cdot r$ & كنترل & \\
\hline \multirow{2}{*}{.$/ 40$} & .19. & $\mid V+/ 9 Y \pm E \varphi / 18$ & $|E q / \triangle A \pm E V|+A$ & تجربي & \multirow{2}{*}{ ايمونو كلوبولين A (ميلى كرم } \\
\hline &.$/ N$ & $|Q N \cdot \Lambda \pm \Delta Q / M|$ & $18 N / A 9 \pm 97 / \circ \Delta$ & كنترل & \\
\hline \multirow{2}{*}{$.1 .1^{\dagger}$} & $+1+1^{\dagger}$ & $\| r / N \in \pm 1 / .+$ & $\| T / W \pm N /$ & تجوبي & \multirow{2}{*}{ (نانوكرم بور ميلى ليت) } \\
\hline &.$/ M F$ & $|r / M| \pm|/| P$ & $\mid Y / A \Delta \pm V \cdot \Delta$ & كتثرل & \\
\hline \multirow{2}{*}{.109} & ( D & $\Delta / \Lambda \Delta \pm V / Q$ & $\Delta / F V \pm V / M$ & تجربى & \multirow{2}{*}{ 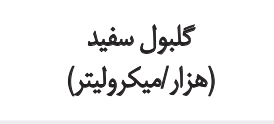 } \\
\hline & $\cdot M$ & $8 / 0 \cdot \pm 1 / 81$ & $g(r) \pm V / \mu r$ & كتثرل & \\
\hline \multirow{2}{*}{ - /Ar } &.$/ \Delta F$ & $M q / v \Delta \pm \mid 1 / T$ & $\Gamma N \cdot \Lambda \pm N V A$ & تجربى & \multirow{2}{*}{ 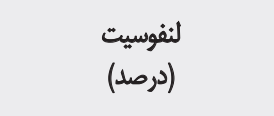 } \\
\hline & $\cdot M$ & $r q / \cdots \pm N T V$ & TNIE $\pm V / \Delta V$ & 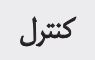 & \\
\hline \multirow{2}{*}{.109} &.$/ O A$ & $\Delta / 91 \pm 1 / 9 Y$ & $\Delta / \Delta \Lambda \pm V / \Delta C$ & تجربي & \multirow{2}{*}{ 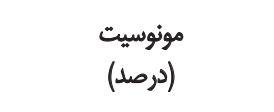 } \\
\hline & 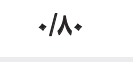 & $f / \cdots \pm 1 / V O$ & $\| / \cdot \wedge \pm V / \cdot \Lambda$ & 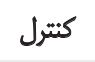 & \\
\hline \multirow{2}{*}{.$|\Delta|$} &.$/ m r$ & $\Delta \Delta / r \Delta \pm I / / M$ & $\Delta H / \cdot A \pm Q / P q$ & تجربى & \multirow{2}{*}{ ن } \\
\hline & $.1 \cdot 1$ & $Q N \cdot \cdot \pm 1 \cdot / M r$ & $\Delta Y / \Delta \cdot \pm V \cdot \Delta$ & كثترل & \\
\hline
\end{tabular}

لنفاوى يا گردش خون نسبت داده مىشود [ [ب]. در تنظيم توليد

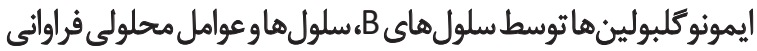

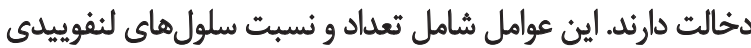

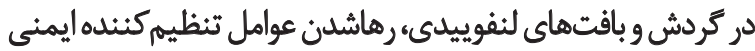

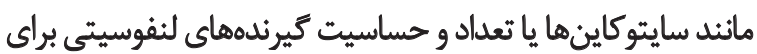

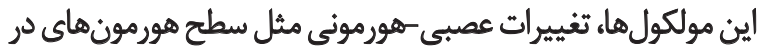

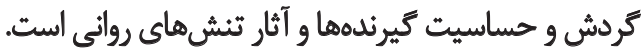
عوامل مذكور ممكن است بهطور موازى با يكديكر عمل كنيند.

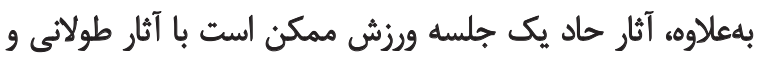

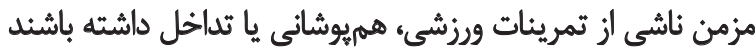

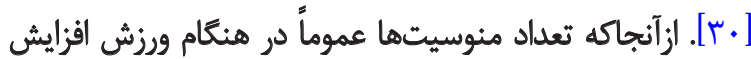

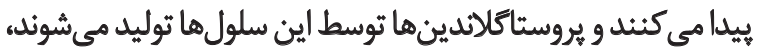

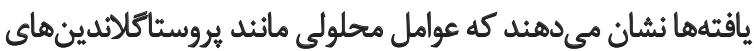

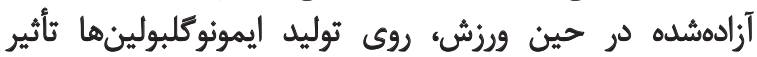

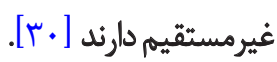

با افزايش سن، سطح طبيعى آنتىبادى موجود در تردش خون
ايمونوكلبولينهاي M و G، بعد از يك فصل اجراى ثمرينات منظمه

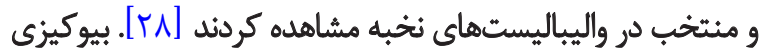

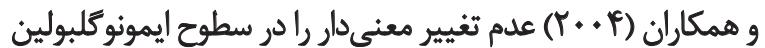

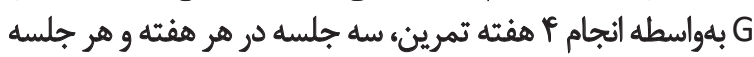

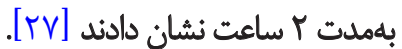

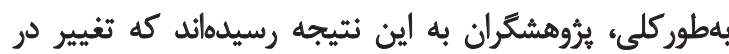

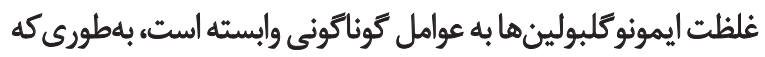

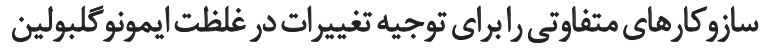

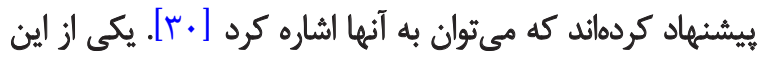

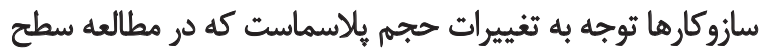

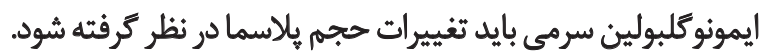

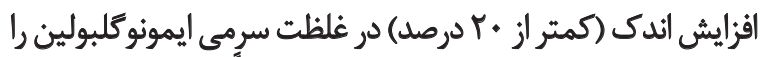
كه يس از ورزشهاى حاد ديده مىشود، عمدتأ مي توان به تغييرات إندات

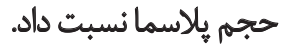

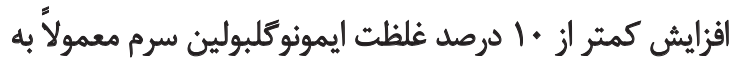
تغييرات روزانه و تبادل ذخيره ايمونوكلبولين خارج عروقى و عروق إنى 
حداكثر اكسيرُن مصرفي انجام شود، افزرايش مي يابد. همجنين ياسخ

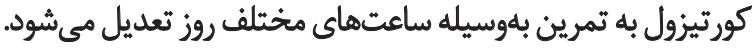
سازوكارهاي مختلفى وجود دارد كه علت افزايش غلظت كورتيزول

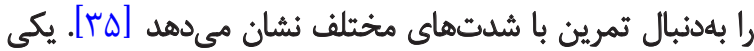

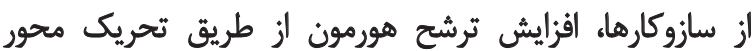

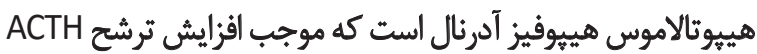

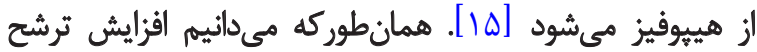
ACTH

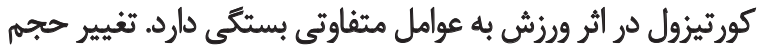

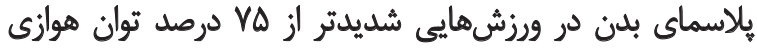

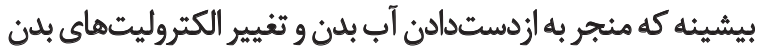

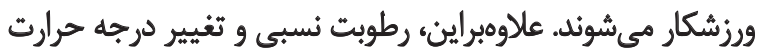

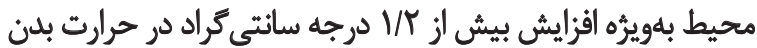

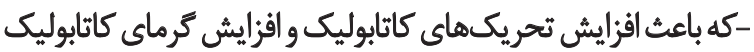

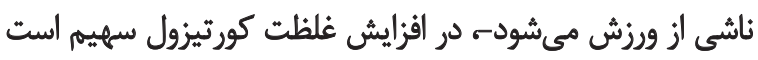

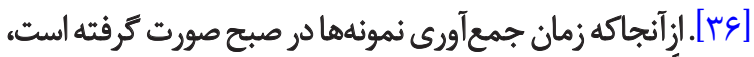

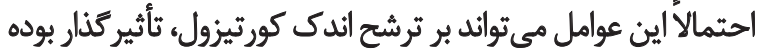
باشد. - ب الآير

\section{نتيجلكيرى نهايى}

با وجود آنكه نتايج يروهشها با هم متفاوت است، يروهشكَران

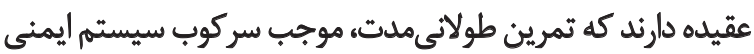

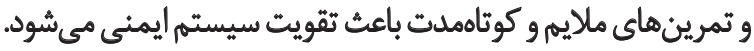

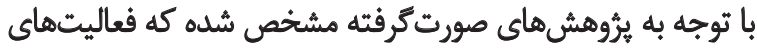

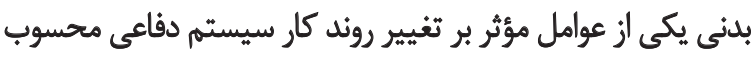

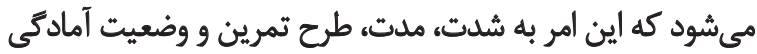
جسمانى، نوع تغذيه، حالات روحى و روانى و عوامل هورمونى افر أراد

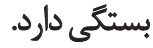

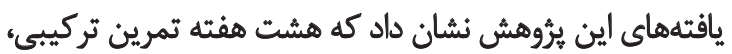

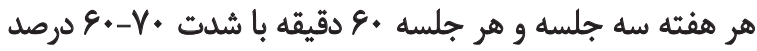

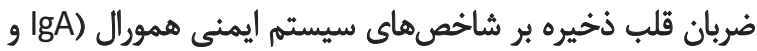

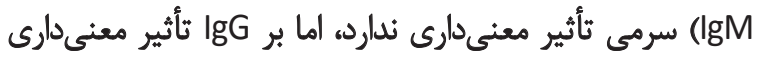

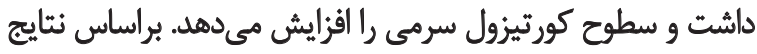

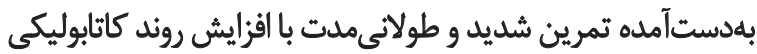

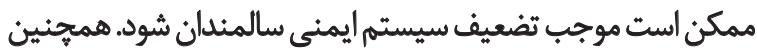

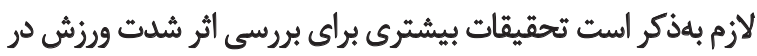

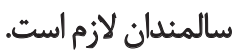

از جمله محدوديثهاي موجود در تحقيق حاضر، تعداد كم بمري

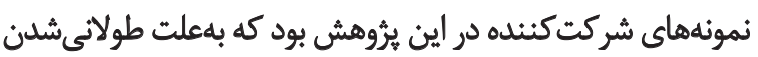

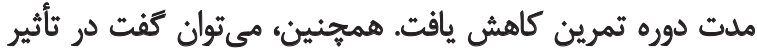

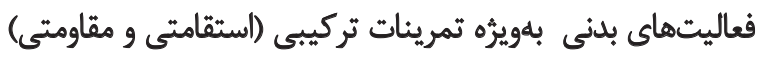
و افزايش سن روى فعاليت ايمنى هومورال سالمندان غيرفيثى ونعال
و واسخ اوليه آنتىبادى كاهش مىيابد. بهنظر ميرسد اين تغييرات

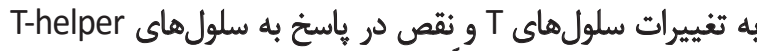

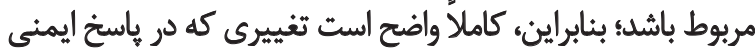

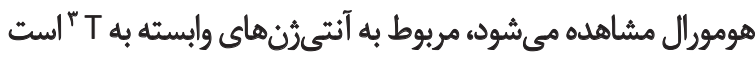

با اين حال، بهنظر ميرسد بعضى از تغييرات بهعلت تحولات

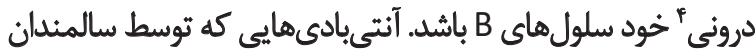

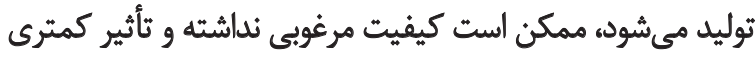

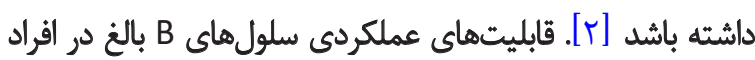

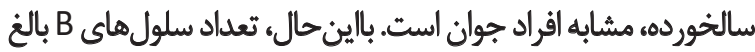

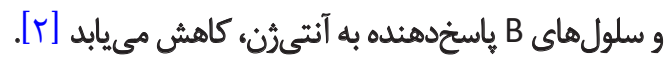
براساس نثايج اين تحقيق، مقادير سطوح كورتيزول سرمى مردان

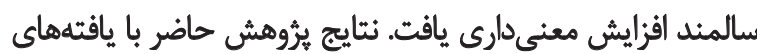

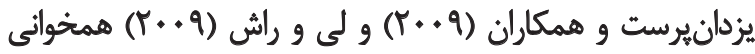

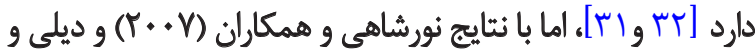

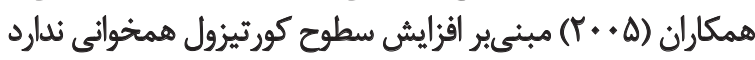

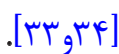

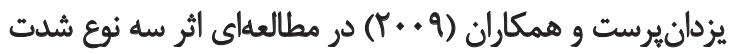

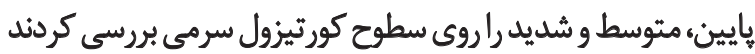

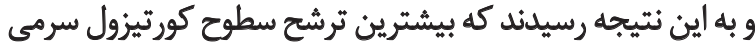

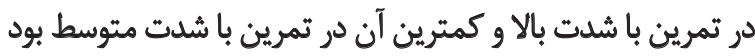

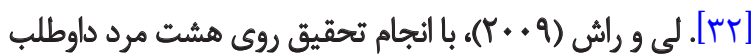

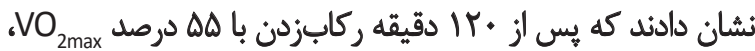

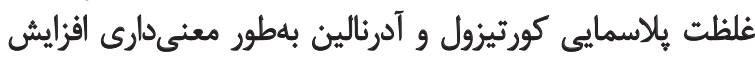

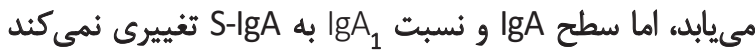

[rا

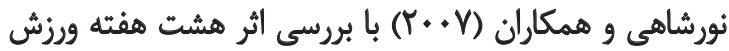

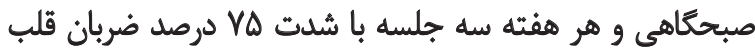

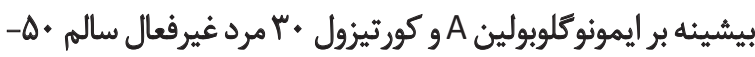

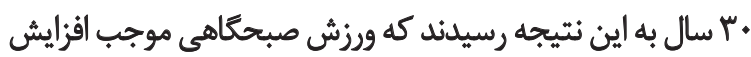

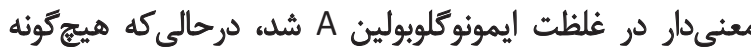

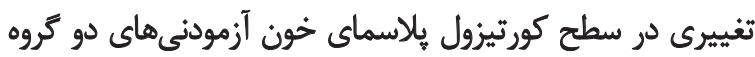

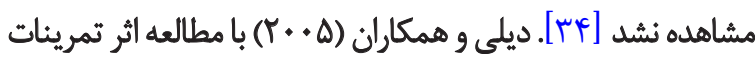

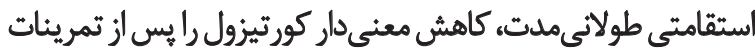

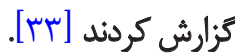

ترشح كورتيزول با توجه به شدت تمرين، متغير است. در باسخ

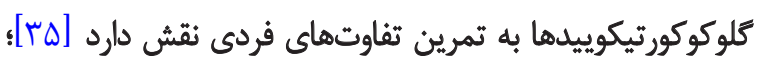

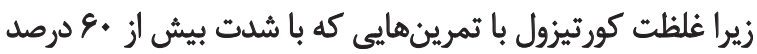

3. T-dependent

4. Intrinsic 


\section{References}

[1] Shimizu K, Sato H, Suga Y, Yamahira S, Toba M, Hamuro K, et al. The effects of Lactobacillus pentosus strain b240 and appropriate physical training on salivary secretory IgA levels in elderly adults with low physical fitness: A randomized, double-blind, placebo-controlled trial. Journal of Clinical Biochemistry and $\mathrm{Nu}-$ trition. 2014; 54(1):61-6.

[2] Laurel T. Advance in exercise immunology. $1^{\text {st }}$ ed. Cincinnati, Ohio: Human Kinetics; 1999.

[3] Nieman D, Henson D, Gusewitch G. Physical activity and immune function in elderly women. Medicine and Science in Sports Exercise. 1993; 25(7):823-31.

[4] Gleeson M. Immune function in sport and exercise. Oxford: Elsevier Health Sciences; 2006.

[5] Houston MS, Silverstein MD, Suman V. Risk factors for 30-day mortality in elderly patients with lower respiratory tract infection: community-based study. Archives of Internal Medicine. 1997; 157(19):2190-5.

[6] Moreira A, Delgado L, Moreira P, Haahtela T. Does exercise increase the risk of upper respiratory tract infections? British Medical Bulletin. 2009; 90(1):111-31.

[7] Moreira A, Mortatti AL, Arruda AF, Freitas CG, de Arruda M, Aoki MS. Salivary IgA response and upper respiratory tract infection symptoms during a 21-week competitive season in young soccer players. Journal of Strength \& Conditioning Research. 2014; 28(2):467-73.

[8] Koch AJ. Immune response to resistance exercise. American Journal of Lifestyle Medicine. 2010; 4(3):244-52.

[9] Laing SJ, Gwynne D, Blackwell J, Williams M, Walters R, Walsh NP. Salivary IgA response to prolonged exercise in a hot environment in trained cyclists. European Journal of Applied Physiology. 2005; 93(5-6):665-71.

[10] Nieman DC, Henson DA, Dumke CL, Lind RH. Relationship between salivary IgA secretion and upper respiratory tract infection following a 160-km race. Journal of Sports Medicine and Physical Fitness. 2006; 46(1):158.

[11] Dimitriou L, Sharp NC, Doherty M. Circadian effects on the acute responses of salivary cortisol and IgA in well trained swimmers. British Journal of Ssports Medicine. 2002; 36(4):260-4.

[12] Djken H, Kelle M, Colpan L, Tumer C, Sermet A. Effect of physical exercise on complement and immunoglobulin levels in wrestlers and sedentary controls. Journal Medical School. 2000; 27(3-4):39-45.

[13] Moughan R, Gleeson M, Greenhaff P. Biochemistry of exercise and training [AA. Gaeeni, MR. Hamedinia, M. Kooshki Jahromi \& M. Fathi, Persian trans]. $1^{\text {st }}$ ed. Tehran: Noorpardazan; 2001.

[14] McGuigan M, Egan A, Foster C. Salivary cortisol responses and perceived exertion during high intensity and low intensity bouts of resistance exercise. Journal of Sport Science and Medicine. 2004; 3(1):8-15.

[15] Mohebbi H, Azizi M, Moradiani H. Effect of 8 Weeks Low and High Intensity Resistance Training on Leukocyte Count, Igg, Cortisol and Lactate Concentration in Untrained Men. World Applied Sciences Journal. 2012; 16(7):949-54.
مطالعات محدودى وجود دارد و نتّايج حاصل از مطالعات نشائكر

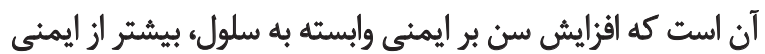

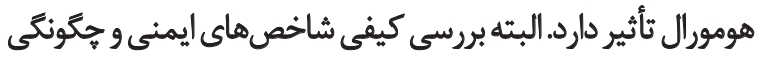
تمرينات مختلف از نظر شدت و حجم تمرين، اهميت زيادى دارئي دارد كه

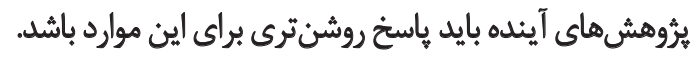

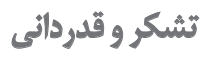

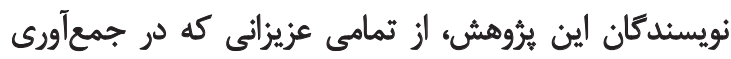

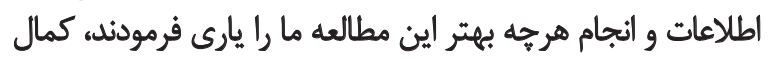
تشكر و قدردانى را اعلام مى هارئد. 
[16] Akimoto T, Kumai Y, Akama T, Hayashi E, Murakami H, Soma $R$, et al. Effects of 12 months of exercise training on salivary secretory IgA levels in elderly subjects. British Journal of Sports Medicine. 2003; 37(1):76-9.

[17] Nehlsen-Cannarella SL, Nieman DC, Balk-Lamberton AJ, Markoff PA, Chritton DB, Gusewitch GA, et al. The effects of moderate exercise training on immune response. Medicine and Science in Sports and Exercise. 1991; 23(1):64-70.

[18] Akbarpour Beni M, Akbari Z, Assarzadeh M, Azizbeigi K. The Effect of Selected Aerobic Training on Serum Immunoglobulin Levels and Testosterone and Cortisol Hormones in Young Men. International Journal of Sport Studies. 2013; 3(9):956-62.

[19] Etemadi H, Nikseresht A. The influence of duration of longdistance road cycling on the amounts of immunoglobulin A \& G in blood of professional cyclists. Advances in Environmental Biology. 2013; 7(9):2105-10

[20] Shephard RJ. Readiness for physical activity. President's Council on Physical Fitness and Sports; 1994.; 1(5):359.

[21] Bijeh N, Hosseini SA, Hejazi K. The effect of aerobic exercise on serum C - reactive protein and leptin levels in untrained middleaged women. Iranian Journal of Public Health. 2012; 41(9):36-41.

[22] Abdolmaleki Z, Saleh Sedghpour B, Bahram A, Abdolmaleki F. Validity and reliability of the physical self-description questionnaire among adolescent girls. Journal of Applied Psychology. 2011; 4(16):42-55

[23] Noushabadi A, Abedi B. Effects of combination training on insulin resistance index and some inflammatory markers in inactive men. Horizon of Medical Sciences. 2012; 18(3):95-105.

[24] Acevedo EO, Starks MA. Exercise Testing and prescription lab manual. Champaign: Human Kinetics; 2003.

[25] Klentrou P, Cieslak T, MacNeil M, Vintinner A, Plyley M. Effect of moderate exercise on salivary immunoglobulin $\mathrm{A}$ and infection risk in humans. European Journal of Applied Physiology. 2002; 87(2):153-8.

[26] Verde TJ, Thomas SG, Moore RW, Shek PA, Shephard RJ. Immune responses and increased training of the elite athlete. Journal of Applied Physiology. 1992; 73(4):1494-9.

[27] Buyukyazi G. Differences in the cellular and humoral immune system between sedentary and endurance-trained elderly males. Science \& Sports. 2004;19(3):130-5.

[28] Crdova A, Sureda A, Tur J, Pons A. Immune response to exercise in elite sportsmen during the competitive season. Journal of Physiological Biochemistry. 2010; 66(1):1-6.

[29] Verde T, Thomas S, Moore R, Shek P, Shephard R. Immune responses and increased training of the elite athlete, immune function in sport and exercise. Journal of Applied Physiology. 2007; 103:693-9.

[30] Mackinnon L, Mackinnon L. Advance in exercise immunology. $3^{\text {rd }}$ ed. Champaign: Human Kinetics; 1999.

[31] Li TL, Rush B. The Effects of Prolonged Strenuous Exercise on Salivary Secretion of IgA Subclasses in Men. International Journal of Sport and Exercise Science. 2009; 1(3):69-74.

[32] Yazdanparast B, Azarbayjani AM, Rasaee MJ, Jourkesh M, Ostojić SM. The effect of different intensity of exercise on salivary steroids concentration in elite girl swimmers. Facta universitatisseries: Physical Education and Sport. 2009; 7(1):69-77.

[33] Daly W, Seeqers C, Rubin D, Hackney A. Relationship between stress hormones and testosterone with prolonged endurance exercise. European Journal of Applied Physiology. 2005; 93(4):375-89.

[34] Nourshahi M, Hovanloo F, Arbabi A. [Effect of Exercise with Moderate Intensity in the Morning on Some Factors of Immune Systems in Adults (Persian)]. Iranian Journal of Endocrinology and Metabolism. 2007; 10(3):241-5.

[35] Kakooei H, Zamanian Ardakani Z, Karimian M, Ayttollahi T. [Twenty four hours circadian cortisol profile in shift work nurses (Persian)]. Armaghan-e Danesh. 2009; 14(1):47-56.

[36] Gleeson M. Mucosal immune responses and risk of respiratory illness in elite athletes. Exercise Immunology Review. 2000; 6:5-42 
